\title{
ON CONTINUABLE RIEMANN SURFACES
}

\author{
NAONDO JIN
}

\section{Introduction}

Let $R$ be a Riemann surface. If there exists a conformal mapping $l$ of $R$ onto a subregion of a Riemann surface $\tilde{R}$, then we call $\tilde{R}$, or more precisely the pair $(\tilde{R}, l)$, an extension of $R$. We often identify $l(R)$ with $R$ and consider $R$ as a subregion of $\tilde{R}$. According to this definition $R$ itself is an extension of $R$. It is called a proper extension if $\tilde{R} \backslash l(R) \neq \emptyset$. A Riemann surface is called continuable if it has proper extensions. A Riemann surface which has no proper extensions is called maximal.

We want to know where the class of all maximal Riemann surfaces has place in the classification theory of Riemann surfaces. We know that if $R$ has a small ideal boundary then $R$ is maximal. For example if $R$ with no planar ends belongs to the class $O_{H D}, O_{K D}$, or $O_{\gamma}$, then $R$ is maximal; see [SO, X.5C].

By a neighborhood of the ideal boundary of $R$ we mean the exterior of a compact set of $R$. We call a connected component $V$ of a neighborhood of the ideal boundary an end if it is not relatively compact. Let $D$ be a simply connected subregion of $R$. Suppose that its relative boundary $\partial D$ consists of a countable number of analytic simple open arcs $\left\{\gamma_{j}\right\}$ such that each $\gamma_{j}$ starts from the ideal boundary and terminates at the ideal boundary, $\gamma_{j} \cap \gamma_{k}=\emptyset$ if $j \neq k$, and $\left\{\gamma_{j}\right\}$ does not accumulate in $R$. Then a Riemann mapping $\phi$ of $D$ onto the unit disc $U$ is continuously extended over $\partial D$ and $\phi(\partial D)$ is a relatively open subset of $\partial U$. We denote by $I$ the complement of $\phi(\partial D)$ with respect to $\partial U$. We call $D$ a disc with crowded ideal boundary if $I$ is totally disconnected and is not an $N_{D}$-set.

In [Sa, Example 2] Sakai showed that there exists a two-sheeted unlimited covering surface of the unit disc which is maximal, using the following characterization of continuable Riemann surfaces:

THEOREM A [Sa, Theorem 4.1]. Let $R$ be a Riemann surface. Then $R$ is continuable if and only if one of the following conditions holds for $R$.

This work is supported by Grant-1n-Aid for Encouragement of Young Scientists (No. 05740112), The Ministry of Education, Science and Culture.

Received March 26, 1998; revised May 28, 1998. 
(a) $R$ has a planar end.

(b) $R$ has a border.

(c) $R$ has a disc with crowded ideal boundary.

As the first step we treat $n$-sheeted unlimited covering surfaces $R$ of the unit disc. In this paper we obtain a sufficient condition of $R$ to be continuable. In [J] we consider sufficient conditions of $R$ to be maximal.

\section{Results}

Let $R$ be an open Riemann surface which possesses the Green function $g_{p_{0}}(p)$. Set $R\left(p_{0}, \alpha\right)=\left\{p \in R ; g_{p_{0}}(p)>\alpha\right\}$ for $\alpha>0$. We denote by $B\left(p_{0}, \alpha\right)$ the first Betti number of $R\left(p_{0}, \alpha\right)$. As is known from Sakai's characterization of continuable Riemann surfaces, we need some informations about the neighborhood of the ideal boundary. First we shall consider a Riemann surface $R$ which satisfies the following condition $(\mathrm{A})$ for some point $p_{0} \in R$ :

$$
\int_{0}^{\frac{1}{2}} B\left(p_{0}, \alpha\right) \frac{d \alpha}{\alpha(\log \alpha)^{2}}<\infty .
$$

We say that $R$ is regular in the sense of the potential theory if $g_{p_{0}}(p)$ tends to 0 as $p$ tends to the ideal boundary of $R$, or equivalently $R\left(p_{0}, \alpha\right)$ is relatively compact for every $\alpha$; see [Ha, p. 84].

Our result is

THEOREM 1. Suppose that $R$ satisfies condition (A). Then $R$ is continuable. Furthermore if $R$ is regular in the sense of the potential theory, then $R$ has a disc with crowded ideal boundary.

Remark. Widom [W] considered a Riemann surface which satisfies the following condition:

$$
\int_{0}^{\infty} B\left(p_{0}, \alpha\right) d \alpha<\infty
$$

A Riemann surface which satisfies the condition (PW) is called of ParreauWidom type. It is easily seen that if $R$ satisfies condition (A), then $R$ is of Parreau-Widom type.

Next let $n \geq 2$ and $R$ be an $n$-sheeted unlimited covering surface of the unit disc $U$ with the projection mapping $\pi$. The pair $(R, \pi)$ is also called a covering surface. Let $\left\{z_{v}\right\}$ be the set of the projection of branch points. We consider a Riemann surface $R$ which satisfies the following condition:

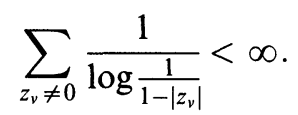


It is clear that condition $\left(\mathrm{A}^{\prime}\right)$ is stronger than the Blaschke condition $\sum\left(1-\left|z_{v}\right|\right)<\infty$. Then as a corollary of Theorem 1 we have

THEOREM 2. Let $n \geq 2$ and $(R, \pi)$ be an $n$-sheeted unlimited covering surface of the unit disc $U$ and $\left\{z_{v}\right\}_{v \geq 1}$ the projection of branch points. If $\left\{z_{v}\right\}$ satisfies condition $\left(\mathrm{A}^{\prime}\right)$, then $R$ is continuable.

Remark. By [Sa, Example 1'] it is known that there is a continuable twosheeted unlimited covering surface of the unit disc such that the derived set of $\left\{z_{v}\right\}$ is equal to $\partial U$ and satisfies, for example, $\sum\left(1-\left|z_{v}\right|\right)=\infty$. Then sufficient conditions of $R$ to be maximal are not obtained by using the absolute values $\left\{\left|z_{v}\right|\right\}$. Thus we can not obtain the characterization of maximality in terms of the absolute values $\left\{\left|z_{v}\right|\right\}$.

It is not known whether condition (PW) is sufficient to be continuable or not.

\section{Condition (A)}

In this section we shall show some properties of a Riemann surface satisfying condition (A). We obtain the next proposition about condition (A) (cf. [Ha, Chapter V]).

Proposition 1. Condition (A) is independent of the choice of $p_{0} \in R$.

Proof. Take any two distinct points $p_{0}$ and $p_{1} \in R$. We may assume that

$$
\int_{0}^{\frac{1}{2}} B\left(p_{0}, \alpha\right) \frac{d \alpha}{\alpha(\log \alpha)^{2}}<\infty .
$$

For sufficiently large $M>0, \overline{R\left(p_{0}, M\right)}=\left\{p \in R ; g_{p_{0}}(p) \geq M\right\}$ and $\overline{R\left(p_{1}, M\right)}$ are mutually disjoint closed discs. There exists $m, 0<m<1$, such that $m g_{p_{1}}(p)<g_{p_{0}}(p)$ on $R \backslash\left(\overline{R\left(p_{0}, M\right)} \cup \overline{R\left(p_{1}, M\right)}\right)$. It follows that $R\left(p_{0}, m \alpha\right) \supset$ $R\left(p_{1}, \alpha\right)$ for $0<\alpha<M$. We know that $R\left(p_{0}, m \alpha\right) \backslash R\left(p_{1}, \alpha\right)$ does not have a compact component. Therefore there is a natural inclusion $H_{1}\left(R\left(p_{1}, \alpha\right)\right) \hookrightarrow$ $H_{1}\left(R\left(p_{0}, m \alpha\right)\right)$ between singular homology groups. So we have $B\left(p_{0}, m \alpha\right) \geq$ $B\left(p_{1}, \alpha\right)$ and

$$
\int_{0}^{\frac{1}{2}} B\left(p_{1}, \alpha\right) \frac{d \alpha}{\alpha(\log \alpha)^{2}} \leq \int_{0}^{\frac{1}{2}} B\left(p_{0}, m \alpha\right) \frac{d \alpha}{\alpha(\log \alpha)^{2}} .
$$

It suffices to show that the right side integral is finite. Since $(\log \alpha)^{2} /(\log \alpha / m)^{2}$ tends to 1 as $\alpha \rightarrow 0$, there exists $K>0$ such that $(\log \alpha)^{2} /(\log \alpha / m)^{2}<K$. Then we have 


$$
\begin{aligned}
\int_{0}^{\frac{1}{2}} B\left(p_{0}, m \alpha\right) \frac{d \alpha}{\alpha(\log \alpha)^{2}} & =\int_{0}^{\frac{m}{2}} B\left(p_{0}, \alpha\right) \frac{d \alpha}{\alpha\left(\log \frac{\alpha}{m}\right)^{2}} \\
& <K \int_{0}^{\frac{1}{2}} B\left(p_{0}, \alpha\right) \frac{d \alpha}{\alpha(\log \alpha)^{2}}<\infty
\end{aligned}
$$

The proof is completed.

For the Green function $g_{p_{0}}(p)$ we define a meromorphic differential $\omega_{p_{0}}=$ $d g_{p_{0}}+i d g_{p_{0}}^{*}$. We say that $q \in R$ is a critical point of $g_{p_{0}}$ if $\operatorname{grad} g_{p_{0}}(q)=0$ or equivalently $\omega_{p_{0}}$ has a zero at $q$. We call the order of zeros of $\omega_{p_{0}}$ at $q$ the degree at the critical point $q$. Obviously the set of critical points is discrete. Then it is at most countable. The following lemma is useful.

Lemma 1. Let $R$ be a regular Riemann surface in the sense of the potential theory. Then $R$ satisfies condition (A) if and only if

$$
\sum_{g_{p_{0}}\left(q_{k}\right)<\frac{1}{2}} \frac{v_{k}}{\log \frac{1}{g_{p_{0}}\left(q_{k}\right)}}<\infty
$$

where $\left\{q_{k}\right\}_{k \geq 1}$ is the set of critical points of $g_{p_{0}}(p)$ and $v_{k}$ is the degree at $q_{k}$. More precisely the following equation holds.

$$
\int_{0}^{\frac{1}{2}} B\left(p_{0}, \alpha\right) \frac{d \alpha}{\alpha(\log \alpha)^{2}}=\frac{B\left(p_{0}, \frac{1}{2}\right)}{\log 2}+\sum_{g_{p_{0}}\left(q_{k}\right)<\frac{1}{2}} \frac{v_{k}}{\log \frac{1}{g_{p_{0}}\left(q_{k}\right)}}
$$

Proof. Denote by $\left\{q_{1}, \ldots, q_{\mu}\right\}$ the set of all critical points in $R\left(p_{0}, \alpha\right)$. First we show that $B\left(p_{0}, \alpha\right)=\sum_{k=1}^{\mu} v_{k}$. We may assume that $\partial R\left(p_{0}, \alpha\right)$ contains no critical points. Consider the double $\hat{R}\left(p_{0}, \alpha\right)$ of $R\left(p_{0}, \alpha\right)$ along $\partial R\left(p_{0}, \alpha\right)$. Let $\psi$ be the anti-conformal automorphism on $\hat{R}\left(p_{0}, \alpha\right)$.

We know that $\hat{\boldsymbol{g}}_{\alpha}=B\left(p_{0}, \alpha\right)$, where $\hat{\boldsymbol{g}}_{\alpha}$ is the genus of $\hat{R}\left(p_{0}, \alpha\right)$. We shall show $\hat{\boldsymbol{g}}_{\alpha}=\sum_{k=1}^{\mu} v_{k}$. On the double $\hat{R}\left(p_{0}, \alpha\right)$ the function $g_{p_{0}}(p)-\alpha$ can be extended symmetrically to a function $\hat{g}(p)$ which is harmonic in $\hat{R}\left(p_{0}, \alpha\right) \backslash$ $\left\{p_{0}, \psi\left(p_{0}\right)\right\}$ and has a singularity of $-\log |z|$ (resp. $\left.\log |z|\right)$ at $p=p_{0}$ (resp. $p=$ $\left.\psi\left(p_{0}\right)\right)$. Then the set of critical points of $\hat{g}$ is $\left\{q_{1}, \ldots, q_{\mu}, \psi\left(q_{1}\right), \ldots, \psi\left(q_{\mu}\right)\right\}$. The meromorphic differential $\omega_{p_{0}}$ has poles of order 1 at $p_{0}$ and $\psi\left(p_{0}\right)$ and zeros of order $v_{k}$ at $q_{k}$ and $\psi\left(q_{k}\right)$. Since $\hat{R}\left(p_{0}, \alpha\right)$ is compact, for every meromorphic differential on it the degree of divisor is equal to $2 \hat{\boldsymbol{g}}_{\alpha}-2$. Then $\operatorname{deg}\left(\omega_{p_{0}}\right)=$ $2 \hat{\boldsymbol{g}}_{\alpha}-2=2 \sum_{k=1}^{\mu} v_{k}-2$. Therefore we have $\hat{\boldsymbol{g}}_{\alpha}=\sum_{k=1}^{\mu} v_{k}$.

In terms of Stieltjes integral for any $\varepsilon>0$ we have 


$$
\begin{aligned}
\sum_{\varepsilon<g_{p_{0}}\left(q_{k}\right)<\frac{1}{2}} \frac{v_{k}}{\log \frac{1}{g_{p_{0}}\left(q_{k}\right)}} & =-\int_{\varepsilon}^{\frac{1}{2}} \frac{1}{\log \frac{1}{\alpha}} d B\left(p_{0}, \alpha\right) \\
& =\int_{\varepsilon}^{\frac{1}{2}} B\left(p_{0}, \alpha\right) d\left(\frac{1}{\log \frac{1}{\alpha}}\right)-B\left(p_{0}, \frac{1}{2}\right) \frac{1}{\log 2}+B\left(p_{0}, \varepsilon\right) \frac{1}{\log \frac{1}{\varepsilon}}
\end{aligned}
$$

Since

$$
0<B\left(p_{0}, \varepsilon\right) \frac{1}{\log \frac{1}{\varepsilon}}<\int_{0}^{\varepsilon} B\left(p_{0}, \alpha\right) d\left(\frac{1}{\log \frac{1}{\alpha}}\right)
$$

we have

$$
\begin{aligned}
\int_{\varepsilon}^{\frac{1}{2}} B\left(p_{0}, \alpha\right) d\left(\frac{1}{\log \frac{1}{\alpha}}\right)-B\left(p_{0}, \frac{1}{2}\right) \frac{1}{\log 2} & \\
& <\sum_{\varepsilon<g_{p_{0}}\left(q_{k}\right)<\frac{1}{2}} \frac{v_{k}}{\log \frac{1}{g_{p_{0}}\left(q_{k}\right)}}<\int_{0}^{\frac{1}{2}} B\left(p_{0}, \alpha\right) d\left(\frac{1}{\log \frac{1}{\alpha}}\right)-B\left(p_{0}, \frac{1}{2}\right) \frac{1}{\log 2}
\end{aligned}
$$

As $\varepsilon \rightarrow 0$, we have the conclusion:

$$
\int_{0}^{\frac{1}{2}} B\left(p_{0}, \alpha\right) \frac{d \alpha}{\alpha(\log \alpha)^{2}}=\frac{B\left(p_{0}, \frac{1}{2}\right)}{\log 2}+\sum_{g_{p_{0}}\left(q_{k}\right)<\frac{1}{2}} \frac{v_{k}}{\log \frac{1}{g_{p_{0}}\left(q_{k}\right)}}
$$

In the general case if $R$ satisfies condition (A), then for every $\alpha>0 B\left(p_{0}, \alpha\right)$ is finite. Hence $R\left(p_{0}, \alpha\right)$ can be considered as a compact bordered Riemann surface with finite punctures. By adding a finite number of points to $R\left(p_{0}, \alpha\right)$ for each $\alpha$ we can construct a regular Riemann surface $\tilde{R}$ in the sense of the potential theory such that $R$ is a proper subregion of $\tilde{R}$ and $E=\tilde{R} \backslash R$ is a discrete set. This $\tilde{R}$ is called a regularization of $R$; see [Ha, pp. 86-87]. Since the Green function for $R$ is the restriction of that for $\tilde{R}$ to $R$, we can prove the following lemma. See Lemma $3 \mathrm{~A}$, Theorem $3 \mathrm{~B}$, and Theorem $3 \mathrm{C}$ in $[\mathrm{Ha}, \mathrm{pp}$. 86-90].

LEMMA 2. Suppose that $R$ satisfies condition (A). Then the regularization $\tilde{R}$ of $R$ also satisfies condition (A). Moreover the following equation holds:

$$
\begin{aligned}
& \int_{0}^{\frac{1}{2}} \tilde{B}\left(p_{0}, \alpha\right) \frac{d \alpha}{\alpha(\log \alpha)^{2}}-\frac{1}{\log 2} \tilde{B}\left(p_{0}, \frac{1}{2}\right) \\
& \quad=\int_{0}^{\frac{1}{2}} B\left(p_{0}, \alpha\right) \frac{d \alpha}{\alpha(\log \alpha)^{2}}-\frac{1}{\log 2} B\left(p_{0}, \frac{1}{2}\right)-\sum_{q \in \tilde{R} \backslash R, \tilde{g}_{p_{0}}(q)<\frac{1}{2}} \frac{1}{\log _{\tilde{g}_{p_{0}}(q)}},
\end{aligned}
$$


where $\tilde{g}_{p_{0}}(q)$ is the Green function of $\tilde{R}$ and $\tilde{B}\left(p_{0}, \alpha\right)$ is the first Betti number of $\tilde{R}\left(p_{0}, \alpha\right)=\left\{p \in \tilde{R} ; \tilde{g}_{p_{0}}(q)>\alpha\right\}$.

Proof. It is easily seen that if $p_{0} \in R$ then $\tilde{g}_{p_{0}}=g_{p_{0}}$ holds in $R$. Since $\tilde{R}\left(p_{0}, \alpha\right) \cap R=R\left(p_{0}, \alpha\right)$, we have $B\left(p_{0}, \alpha\right)-\tilde{B}\left(p_{0}, \alpha\right)$ is equal to the cardinal number of $\tilde{R}\left(p_{0}, \alpha\right) \backslash R\left(p_{0}, \alpha\right)$. Thus for any $\varepsilon>0$ we have

$$
\int_{\varepsilon}^{\frac{1}{2}} \frac{1}{\log \frac{1}{\alpha}} d B\left(p_{0}, \alpha\right)-\int_{\varepsilon}^{\frac{1}{2}} \frac{1}{\log \frac{1}{\alpha}} d \tilde{B}\left(p_{0}, \alpha\right)=-\sum_{q \in \tilde{R}\left(p_{0}, \varepsilon\right) \backslash R\left(p_{0}, \varepsilon\right), \tilde{g}_{p_{0}}(q)<\frac{1}{2}} \frac{1}{\log _{\frac{g_{p_{0}}}{(q)}}},
$$

and

$$
\begin{aligned}
\int_{\varepsilon}^{\frac{1}{2}} \tilde{B}\left(p_{0}, \alpha\right) \frac{d \alpha}{\alpha(\log \alpha)^{2}}-\int_{\varepsilon}^{\frac{1}{2}} B\left(p_{0}, \alpha\right) \frac{d \alpha}{\alpha(\log \alpha)^{2}} \\
=-\frac{1}{\log 2}\left\{B\left(p_{0}, \frac{1}{2}\right)-\tilde{B}\left(p_{0}, \frac{1}{2}\right)\right\}+\frac{1}{\log \frac{1}{\varepsilon}}\left\{B\left(p_{0}, \varepsilon\right)-\tilde{B}\left(p_{0}, \varepsilon\right)\right\} \\
\quad-\sum_{q \in \tilde{R}\left(p_{0}, \varepsilon\right) \backslash R\left(p_{0}, \varepsilon\right), \tilde{g}_{p_{0}}(q)<\frac{1}{2}} \frac{1}{\frac{\log \frac{1}{\tilde{g}_{p_{0}}(q)}}{}} .
\end{aligned}
$$

As $\varepsilon \rightarrow 0$ we have the required conclusion.

\section{Proof of Theorem 2}

Before proving Theorem 1 we shall show Theorem 2 by using Theorem 1 . We prepare the next lemma.

Lemma 3. Let $R$ and $R^{\prime}$ be Riemann surfaces. Suppose that there are regular subregions $\Omega$ and $\Omega^{\prime}$ of $R$ and $R^{\prime}$, respectively, and a conformal mapping $\psi$ of $R \backslash \Omega$ onto $R^{\prime} \backslash \Omega^{\prime}$ such that $\psi(\partial \Omega)=\partial \Omega^{\prime}$. Then $R$ is continuable if and only if $R^{\prime}$ is continuable.

Proof. It is enough to show that if $R$ is continuable then $R^{\prime}$ is continuable. Let $(\tilde{R}, l)$ be a proper extension of $R$. Then $l(\Omega)$ is a regular subregion of $\tilde{R}$. Consider a set $(\tilde{R} \backslash l(\Omega)) \cup \Omega^{\prime}$ and identify a point $p \in \partial l(\Omega)$ with $\psi \circ l^{-1}(p) \in$ $\partial \Omega^{\prime}$. Then we obtain an extension $\tilde{R}^{\prime}$ of $R^{\prime}$. Since $\tilde{R} \backslash l(R) \neq \emptyset, \tilde{R}^{\prime}$ is a proper extension of $R^{\prime}$ and hence $R^{\prime}$ is continuable.

Proof of Theorem 2. Let $T(z)$ be a Möbius transformation which preserves the unit disc. Then $(R, T \circ \pi)$ is also an $n$-sheeted unlimited covering surface of the unit disc. First we shall check that $(R, T \circ \pi)$ satisfies the condition $\left(\mathrm{A}^{\prime}\right)$. We know that $T(z)$ is of the form $(z-\alpha) /(1-\bar{\alpha} z)$ for some $\alpha \in U$. By 
the Schwarz-Pick lemma we deduce that

$$
\frac{1-|\alpha|^{2}}{2(1+|\alpha|)^{2}} \leq \frac{1-|T(z)|}{1-|z|} \leq \frac{2\left(1-|\alpha|^{2}\right)}{(1-|\alpha|)^{2}} .
$$

Since the set $\left\{z_{v}\right\}$ of the projection of branch points satisfies

$$
\sum_{z_{v} \neq 0} \frac{1}{\log \frac{1}{1-\left|z_{v}\right|}}<\infty
$$

we have

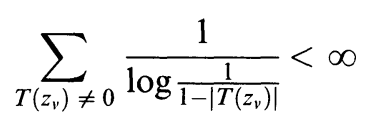

and hence $(R, T \circ \pi)$ satisfies the condition $\left(\mathrm{A}^{\prime}\right)$.

If $0 \in\left\{z_{v}\right\}_{v>1}$, we consider a covering surface $(R, T \circ \pi)$ instead of $(R, \pi)$ so that $0 \notin\left\{T\left(z_{v}\right)\right\}_{v \geq 1}$. Hence we may assume $0 \notin\left\{z_{v}\right\}$. Since there is $\rho>0$ such that $\left\{z_{v}\right\} \cap\{|z| \leq \rho\}=\emptyset, \quad \pi^{-1}(\{|z|<\rho\})$ consists of $n$ components, $B_{1}, \ldots, B_{n}$. Set $B_{j} \cap \pi^{-1}(l)=l_{j}$, where $l$ is the closed interval $[0, \rho / 2]$ on the real axis, and $B_{j}^{\prime}=B_{j} \backslash l_{j}$. We identify the upper edge of $B_{j}^{\prime}$ by the lower edge of $B_{j+1}^{\prime}, j=1, \ldots, n-1$, and the upper edge of $B_{n}^{\prime}$ by the lower edge of $B_{1}^{\prime}$. Then we obtain a new $n$-sheeted unlimited covering surface $R_{1}$ of the unit disc with the natural projection mapping $\pi_{1}$ such that $p_{0}=\pi_{1}^{-1}(0)$ is a branch point of degree $n-1$ and there is a conformal mapping $\psi$ of $R \backslash \pi^{-1}(\{|z|<\rho\})$ onto $R_{1} \backslash$ $\pi_{1}^{-1}(\{|z|<\rho\})$ with $\pi=\pi_{1} \circ \psi$. By Lemma 3 it suffices to show that $R_{1}$ is continuable.

The Green function $g_{p_{0}}(p)$ on $R_{1}$ is represented as $(1 / n) \log \left(1 /\left|\pi_{1}(p)\right|\right)$ and $\left\{\pi_{1}^{-1}\left(z_{v}\right)\right\}_{v \geq 1}$ contains the set of all critical points. It is clear that $R_{1}$ is a regular Riemann surface in the sense of the potential theory. Note that

$$
\sum_{g_{p_{0}}\left(q_{k}\right)<\frac{1}{2}} \frac{v_{k}}{\log \frac{1}{g_{p_{0}}\left(q_{k}\right)}} \leq(n-1) \sum_{\frac{1}{n} \log \frac{1}{\left|z_{v}\right|}<\frac{1}{2}} \frac{1}{\log \frac{1}{\frac{-1}{n} \log \left|z_{v}\right|}}
$$

where $\left\{q_{k}\right\}_{k \geq 1}$ is the set of critical points of $g_{p_{0}}(p)$ and $v_{k}$ is the degree at $q_{k}$. Since $1-\left|z_{v}\right|$ and $-\log \left|z_{v}\right|$ are comparable with each other, $R_{1}$ satisfies condition (A) by Lemma 1 . Hence $R_{1}$ is continuable by Theorem 1 . This completes the proof.

\section{Proof of Theorem 1}

Proof of Theorem 1. By Lemma 2 we may assume that $R$ is regular in the sense of the potential theory. We say an $\operatorname{arc} \gamma$ is a Green arc if $\operatorname{grad} g_{p_{0}} \neq 0$ on 
$\gamma$ and $d g_{p_{0}}^{*}=0$ along $\gamma$ hold. A maximal Green arc will be called a Green line. For sufficiently large $M>0, \overline{R\left(p_{0}, M\right)}$ is a closed disc. In fact the function $\Phi(p)=\exp \left(-g_{p_{0}}(p)-i g_{p_{0}}^{*}(p)\right)$ conformally maps $\overline{R\left(p_{0}, M\right)}$ onto $\left\{|z| \leq e^{-M}\right\}$. For every $\theta \in[0,2 \pi)$ there exists only one Green line $L(\theta)$ which issues from $p_{0}$ and passes through $\Phi^{-1}\left(e^{-M+i \theta}\right)$. A Green line $L(\theta)$ issuing from $p_{0}$ has at most two end points. One is obviously $p_{0}$. In case that $L(\theta)$ tends to the ideal boundary of $R$, we call it regular. In the other case $L(\theta)$ terminates at a critical point of $g_{p_{0}}$. It will be called critical. It is known that $2\left(v_{k}+1\right)$ Green lines meet at a critical point $q_{k}$ of degree $v_{k}$. It is easily seen that at most $v_{k}+1$ critical Green lines $L(\theta)$ issuing from $p_{0}$ terminates at $q_{k}$. We may assume that just $v_{k}+1$ critical Green lines issuing from $p_{0}$ terminate at $q_{k}$. Then there exist $\theta_{k}^{(1)}, \ldots, \theta_{k}^{\left(v_{k}+1\right)}$ in $[0,2 \pi)$ such that $L\left(\theta_{k}^{(1)}\right), \ldots, L\left(\theta_{k}^{\left(v_{k}+1\right)}\right)$ are these critical Green lines. Set $\Omega\left(p_{0}\right)=\left\{p_{0}\right\} \cup \bigcup_{\theta \in[0,2 \pi)} L(\theta)$. Then $\Omega\left(p_{0}\right)$ is a simply connected subregion of $R$, which is called the Green star region. The function $\Phi(p)$ is also a conformal mapping of $\Omega\left(p_{0}\right)$. For our later use we shall consider $\Omega^{\prime}\left(p_{0}\right)=\Omega\left(p_{0}\right) \backslash L(\pi)$. For simplicity let us set $g_{p_{0}}\left(q_{k}\right)=v_{k}$. The function $\Phi(p)$ maps $\Omega^{\prime}\left(p_{0}\right)$ onto a radial slit disc $D$, that is,

$$
D=\Phi\left(\Omega^{\prime}\left(p_{0}\right)\right)=U_{z} \backslash\left(\bigcup_{k=1}^{\infty}\left(l_{k}^{(1)} \cup \cdots \cup l_{k}^{\left(v_{k}+1\right)}\right) \cup(-1,0]\right),
$$

where $U_{z}=\{|z|<1\}$ and $l_{k}^{(j)}=\left\{\rho e^{i \theta} ; e^{-v_{k}} \leq \rho<1, \theta=\theta_{k}^{(j)}\right\}$. By the Riemann mapping $\phi$ the simply connected region $D$ is mapped onto the upper half plane $H=\{\Im \zeta>0\}$. We may assume that $U_{\zeta}=\{|\zeta|<1\}$ contains $I=\partial H \backslash\left(\bigcup_{k=1}^{\infty} \phi\left(l_{k}^{(1)} \cup \cdots \cup l_{k}^{\left(v_{k}+1\right)}\right) \cup \phi((-1,0])\right)$.

On the other hand the mapping $w=\psi(z)=-i \log z$ maps $D$ into a half strip domain $\{w ;-\pi<\mathfrak{R} w<\pi, \mathfrak{I} w>0\}$. Set $\tilde{D}=\psi(D)$ and $\tilde{l}_{k}^{(j)}=\psi\left(l_{k}^{(j)}\right)$. Then $\tilde{l}_{k}^{(j)}=\left\{w=u+i v ; u=\theta_{k}^{(j)}, 0<v \leq v_{k}\right\}$. We may prove Theorem 1 only when the set $I$ is totally disconnected, that is, every point $\beta \in I$ is a boundary component of $\hat{\mathbf{C}} \backslash I$. Since $R$ satisfies also condition (PW), by Theorem on p. 90 of $[\mathrm{Ha}]$

$$
\int_{0}^{\infty} B\left(p_{0}, \alpha\right) d \alpha=\sum_{k} v_{k} v_{k}<\infty
$$

holds. The length of $l_{k}^{(j)}$ 1s equal to $1-e^{-v_{k}}$ and hence it is comparable with $v_{k}$. Thus we have $\sum_{J, k}\left(\right.$ length of $\left.l_{k}^{(j)}\right)=\sum_{k} v_{k}\left(1-e^{-v_{k}}\right)<\infty$. Then we consider $D$ as a Jordan domain bounded by a rectifiable Jordan curve. It is easily seen that two boundary elements are defined over every point of $\bar{l}_{k}^{(j)}=$ $\left\{\rho e^{i \theta} ; e^{-v_{k}}<\rho \leq 1, \theta=\theta_{k}^{(j)}\right\}$ and one boundary element is defined over $e^{i \theta} \in \partial U_{z}$, $\theta \neq \pi, \theta_{k}^{(j)}$.

Recall two general facts about exceptional sets. Let $E$ be a totally disconnected compact set in the unit disc $U$. 
FACT 1 ([SO, XI. 3E]). For a set $E$ on a rectifiable Jordan curve, $E \in N_{D}$ and $E \in N_{S B}$ are equivalent.

For an arbitrary point $\beta \in E$ denote by $\Gamma_{\beta}$ the family of locally rectifiable closed curves in $U \backslash E$ which separates $\beta$ from $\partial U$.

FACT 2 (Characterization of $N_{S B}$-sets by extremal length [SN, II. 8]). $\quad E \in N_{S B}$ if and only if every boundary component $\beta$ is weak, that is, the extremal length $\lambda\left(\Gamma_{\beta}\right)=0$.

If we prove that $I$ is not $N_{D}$-set, then $\Omega^{\prime}\left(p_{0}\right)$ is a disc with crowded ideal boundary. By Facts 1 and 2 it suffices to show that there exists a point $\beta_{0} \in I$ such that $\lambda\left(\Gamma_{\beta_{0}}\right)>0$.

Let $\Gamma_{\beta}^{+}$be the family of locally rectifiable arcs $\gamma$ on $H \cap U_{\zeta}$ each of which connects $(-1, \beta) \backslash I$ and $(\beta, 1) \backslash I$. By the symmetry of $U_{\zeta}$, it is easily seen that $\lambda\left(\Gamma_{\beta}\right)=2 \lambda\left(\Gamma_{\beta}^{+}\right)$. Since extremal length is invariant under any conformal mapping, $\lambda\left(\Gamma_{\beta}^{+}\right)=\lambda\left(\left(\psi \circ \phi^{-1}\right)\left(\Gamma_{\beta}^{+}\right)\right)$. By Carathéodory's theorem $\psi \circ \phi^{-1}$ is extended continuously over $\partial \tilde{D}$. One can see that the family $\left(\psi \circ \phi^{-1}\right)\left(\Gamma_{\beta}^{+}\right)$ consists of locally rectifiable arcs $\gamma$ in $\tilde{D}$ which connects some $\tilde{l}_{k}^{(j)}$ in the right of $b$ and another $\tilde{l}_{m}^{\left(j^{\prime}\right)}$ in the left of $b$, where $b=\left(\psi \circ \phi^{-1}\right)(\beta) \in[-\pi, \pi]$.

Set $f(x)=x \log (1 / x)$ for $x \in(0, \infty)$. Remark that $0<f(x)=x \log (1 / x) \leq 1 / e$ if $x \in(0,1), f(x) \leq 0$ if $x \geq 1$, and $\lim _{x \rightarrow 0} f(x)=0$. Since $R$ satisfies condition (A), by Lemma 1

$$
\begin{aligned}
\sum_{v_{k}<\frac{1}{2}}\left(v_{k}+1\right) \frac{v_{k}}{f\left(v_{k}\right)} & =\sum_{v_{k}<\frac{1}{2}}\left(v_{k}+1\right) \frac{1}{\log \frac{1}{v_{k}}} \\
& \leq \sum_{v_{k}<\frac{1}{2}} 2 v_{k} \frac{1}{\log \frac{1}{v_{k}}}<\infty
\end{aligned}
$$

Hence there exists $k_{0} \in N$ such that $v_{k}<\frac{1}{2}$ if $k \geq k_{0}$ and

$$
\sum_{k=k_{0}}^{\infty}\left(v_{k}+1\right) \frac{v_{k}}{f\left(v_{k}\right)}<\pi
$$

holds.

First we treat the case $k_{0}=1$. Consider the intervals

$$
\left[\theta_{k}^{(j)}-\frac{v_{k}}{f\left(v_{k}\right)}, \theta_{k}^{(j)}+\frac{v_{k}}{f\left(v_{k}\right)}\right]\left(j=1, \ldots, v_{k}+1, k=1,2, \ldots\right)
$$

on the $u$-axis and denote them by $J_{k}^{(j)}$. Then we have 


$$
\begin{aligned}
m_{1}\left(\bigcup_{k=1}^{\infty}\left(J_{k}^{(1)} \cup \cdots \cup J_{k}^{\left(v_{k}+1\right)}\right)\right) & \leq \sum_{k=1}^{\infty} \sum_{j=1}^{v_{k}+1} m_{1}\left(J_{k}^{(j)}\right) \\
& =2 \sum_{k=1}^{\infty}\left(v_{k}+1\right) \frac{v_{k}}{f\left(v_{k}\right)} \\
& <2 \pi,
\end{aligned}
$$

where $m_{1}$ is 1-dimensional Lebesgue measure. Hence $(-\pi, \pi) \backslash \bigcup_{k=1}^{\infty}\left(J_{k}^{(1)} \cup \ldots\right.$ $\left.\cup J_{k}^{\left(v_{k}+1\right)}\right) \neq \emptyset$. Take $b_{0} \in(-\pi, \pi) \backslash \bigcup_{k=1}^{\infty}\left(J_{k}^{(1)} \cup \cdots \cup J_{k}^{\left(v_{k}+1\right)}\right)$. Set $\beta_{0}=\left(\phi \circ \psi^{-1}\right)\left(b_{0}\right)$ $\in I$. We shall show that $\lambda\left(\Gamma_{\beta_{0}}^{+}\right)>0$ or equivalently $\lambda\left(\left(\psi \circ \phi^{-1}\right)\left(\Gamma_{\beta_{0}}^{+}\right)\right)>0$. We define a linear density $\rho_{k}^{(j)}(w)$ on the annulus $A_{k}^{(j)}=\left\{v_{k}<\left|w-\theta_{k}^{(j)}\right|<v_{k} / f\left(v_{k}\right)\right\}$ as follows:

$$
\rho_{k}^{(j)}(w)= \begin{cases}\frac{1}{\left(\log \frac{1}{f\left(v_{k}\right)}\right)\left|w-\theta_{k}^{(j)}\right|} & \text { if } w \in A_{k}^{(j)} \\ 0 & \text { otherwise. }\end{cases}
$$

Set $\rho(w)=\sup _{j, k} \rho_{k}^{(j)}(w)$. Since $b_{0} \notin\left\{J_{k}^{(j)} \cup J_{m}^{\left(j^{\prime}\right)}\right.$ and every $\gamma \in\left(\psi \circ \phi^{-1}\right)\left(\Gamma_{\beta_{0}}^{+}\right)$ connects two slits $\tilde{l}_{k}^{(j)}$ and $\tilde{l}_{m}^{\left(j^{\prime}\right)}$ which lie on each side of $b_{0}$, a subarc of $\gamma$ connects the inner and the outer circles of $A_{k}^{(j)}$. Hence

$$
\begin{aligned}
\int_{\gamma} \rho(w)|d w| \geq \int_{\gamma} \rho_{k}^{(j)}(w)|d w| & \geq \int_{v_{k}}^{\frac{v_{k}}{f\left(v_{k}\right)}} \frac{1}{\log \frac{1}{f\left(v_{k}\right)}} \frac{1}{r} d r \\
& =\frac{1}{\log \frac{1}{f\left(v_{k}\right)}}\left(\log \frac{v_{k}}{f\left(v_{k}\right)}-\log v_{k}\right) \\
& =1
\end{aligned}
$$

or $\rho(w)$ is admissible for $\left(\psi \circ \phi^{-1}\right)\left(\Gamma_{\beta_{0}}^{+}\right)$. By the definition of extremal length

$$
\lambda\left(\left(\psi \circ \phi^{-1}\right)\left(\Gamma_{\beta_{0}}^{+}\right)\right) \geq \frac{1}{\iint \rho^{2}(w) d u d v} .
$$

We have

$$
\begin{aligned}
\iint \rho^{2}(w) d u d v & \leq \sum_{k=1}^{\infty} \sum_{j=1}^{v_{k}+1} \iint_{A_{k}^{(J)}}\left\{\rho_{k}^{(j)}(w)\right\}^{2} d u d v \\
& =\sum_{k=1}^{\infty} \sum_{j=1}^{v_{k}+1} \int_{0}^{2 \pi} d \theta \int_{v_{k}}^{\frac{v_{k}}{f\left(v_{k}\right)}} \frac{1}{\left(r \log \frac{1}{f\left(v_{k}\right)}\right)^{2}} r d r
\end{aligned}
$$




$$
\begin{aligned}
& =2 \pi \sum_{k=1}^{\infty}\left(v_{k}+1\right) \frac{1}{\log \frac{1}{f\left(v_{k}\right)}} \\
& =2 \pi \sum_{k=1}^{\infty}\left(v_{k}+1\right) \frac{1}{\log \frac{1}{v_{k}}} \frac{1}{1-f\left(\frac{1}{\log \frac{1}{v_{k}}}\right)} \\
& \leq \frac{2 \pi e}{e-1} \sum_{k=1}^{\infty}\left(v_{k}+1\right) \frac{1}{\log \frac{1}{v_{k}}} .
\end{aligned}
$$

This is finite by $(*)$. Therefore $\lambda\left(\Gamma_{\beta_{0}}^{+}\right)>0$.

In case of $k_{0}>1$ we can take $b_{0} \in(-\pi, \pi) \backslash \bigcup_{k=k_{0}}^{\infty}\left(J_{k}^{(1)} \cup \ldots \cup J_{k}^{\left(v_{k}+1\right)}\right)$ as above. Set $\beta_{0}=\left(\psi \circ \phi^{-1}\right)\left(b_{0}\right)$. We shall define a subfamily $\Gamma_{1}$ of $\left(\psi \circ \phi^{-1}\right)\left(\Gamma_{\beta_{0}}^{+}\right)$ as follows:

$$
\Gamma_{1}=\left\{\gamma \in\left(\psi \circ \phi^{-1}\right)\left(\Gamma_{\beta_{0}}^{+}\right) ; \gamma \text { connects some } \tilde{l}_{k}^{(j)} \text { and } \tilde{l}_{m}^{\left(j^{\prime}\right)} \text { for } k, m<k_{0}\right\} \text {. }
$$

Set $\Gamma_{2}=\left(\psi \circ \phi^{-1}\right)\left(\Gamma_{\beta_{0}}^{+}\right) \backslash \Gamma_{1}$. It is known that

$$
\frac{1}{\lambda\left(\left(\psi \circ \phi^{-1}\right)\left(\Gamma_{\beta_{0}}^{+}\right)\right)} \leq \frac{1}{\lambda\left(\Gamma_{1}\right)}+\frac{1}{\lambda\left(\Gamma_{2}\right)} .
$$

One can easily show that $\lambda\left(\Gamma_{1}\right)>0$. Define a linear density $\rho_{k}^{(j)}(w), j=$ $1, \ldots, v_{k}+1, k \geq k_{0}$ and $\rho(w)=\sup _{j, k \geq k_{0}} \rho_{k}^{(j)}(w)$ as above. Since $\rho(w)$ is admissible for $\Gamma_{2}$, we deduce that $\lambda\left(\Gamma_{2}\right)>0$. This completes the proof.

Remark. By this proof it follows that for almost all $b \in(-\pi, \pi)$ with respect to the Lebesgue measure the boundary component $\left(\phi \circ \psi^{-1}\right)(b)$ is not weak.

\section{REFERENCES}

[Ha] Hasumi, M., Hardy Classes on Infinitely Connected Riemann Surfaces, Lecture Notes in Math., 1027, Springer, 1983.

[J] JiN, N., On maximality of two-sheeted unlimited coverıng surfaces of the unit disc, to appear in J. Math. Kyoto Univ.

[Sa] SAKAI, M., Continuations of Riemann surfaces, Canad. J. Math., 44 (1992), 357-367

[SN] Sario, L. AND M. NaKaI, Classification Theory of Riemann Surfaces, Springer, 1970.

[SO] Sario, L. and K. Oikawa, Capacity Functions, Springer, 1969.

[W] Widom, H., $\mathscr{H}_{p}$ sections of vector bundles over Riemann surfaces, Ann. of Math., 94 (1971), 304-324.

[Y] Yoshida, M., The method of orthogonal decomposition for differentials on open Riemann surfaces, J. Scı. Hiroshıma Unıv. Ser. A-I Math., 32 (1968), 181-210. 
Department of Mathematics

GAKUSHUIN UNIVERSITY

1-5-1 Mejiro, Toshima-KU

TOKYO 171-8588

JAPAN 